\title{
ANÁLISIS DE UNA MICROTRANSECCIÓN RELACIONADA CON LA PROFUNDIDAD DEL SUELO
}

\author{
por \\ R. Rodríguez González, ${ }^{1}$ A. Puerto, ${ }^{1}$ \\ M. Rico ${ }^{1}$ y J. M. Gómez Gutiérrez ${ }^{1}$
}

\section{SUMMARY}

\section{ANALYSIS OF MICROTRANSECTION AS RELATED TO SOIL DEPTH}

The microheterogeneity of the environment is frecuently related to species distribution. In this study a short distance transection is analysed using soil depth as a basis of differentiation. It is concluded that there exists a niche accomodation of species related to soil depth, althoug the same could also be influenced by other factors like:

a) The distance from rock outcrops.

b) Small changes in soil depth, when soil depth is below $20 \mathrm{~cm}$.

c) Rapid changes of soil depth that determine shifts of species.

d) The existance of transition zones prior to the formation of a typical grassland.

\section{RESUMEN}

La microheterogeneidad del medio se relaciona con frecuencia con la distribución de las especies. En este trabajo se estudia una transección de corto recorrido, tomando como base de diferenciación la profundidad del suelo. Se concluye que hay una acomodación del nicho de las especies de acuerdo con la misma, aunque también pueden influir otras causas, tales como:

a) El alejamiento de los afloramientos rocosos.

b) Pequeños cambios en la profundidad, cuando ésta es inferior a $20 \mathrm{~cm}$.

c) Los cambios rápidos de profundidad, que determinan desplazamientos de las especies.

d) La existencia de zonas de transición, antes de que se alcance el pastizal típico.

\section{INTRODUCCION}

Factores ambientales y tolerancia de las especies son causas y efectos profusamente ligados. Existe una visión macroscópica acerca de la forma en que se relacionan los taxones vegetales y su medio, pero bajo un punto de vista tan amplio quedan supeditadas distribuciones más finas, de detalle, que tienen importancia en cuanto permiten explicar las aptitudes de

(1) Departamento de Ecologia. Facultad de Biologia. Universidad de Salamanca. 
las especies en relación con cambios mínimos de los factores ambientales predomiinantes. Se trata de una expresión de los parámetros del nicho ecológico, afinados al grado máximo, lo que posibilita encuadrar a las especies dentro de límites muy estrictos.

La intensidad de los cambios puede ser verdaderamente notable, por ejemplo en función de la microtopografía, efecto señalado por BAZZAZ (1968) en áreas recientemente labradas. Otras situaciones también resaltan diferencias debidas a escasos centímetros de desnivel (SNAYDON, 1962; FREIJSEN, 1967; ZEDLER y ZEDLER, 1969; GASCO et al., 1979), lo que indica un gran poder de acomodación por parte de los elementos que integran la comunidad.

En la parte central de la provincia de Salamanca, hacia el norte, predomina el encinar castellano (Quercus rotundifolia Lam.), desarrollado en clima termomediterráneo acentuado sobre suelos de tierras pardas con abundantes afloramientos graníticos. Es precisamente la circunstancia de que la roca se encuentre en superficie, a pocos centímetros de profundidad en muchos casos, la que nos ha llevado a emprender el estudio de las relaciones entre vegetación y potencia edáfica, planteado en forma de microgradientes. Una recopilación general de las distintas microtransecciones realizadas ya ha sido puesta de manifiesto por PUERTO et al. (1981). En el presente trabajo pretendemos llevar a cabo una revisión precisa de la transección de mayor longitud $(40 \mathrm{~m})$ incluída en la publicación precedente, a la que se designa con la letra $\mathrm{D}$, la cual presenta caracteres significativos respecto al problema que nos ocupa. Situada en la dehesa de Berrocal de la Espinera (820 m.s.m.; coordenadas U.T.M.: 29T-QF52.37), constituye un enclave representativo de toda la zona circundante.

\section{METODOS}

La vegetación (fanerógamas) fue muestreada de modo continuo, con unidades cuadradas de $10 \mathrm{~cm}$. de lado seriadas una a continuación de otra. A efectos prácticos, y para evitar en lo posible la intervención del azar, dichas unidades se sumaron de cinco en cinco, lo que proporciona un total de 80 rectángulos de muestreo para los cuarenta metros de recorrido.

La primera unidad se sitúa en la proximidad de un afloramiento granítico, siguiéndose en línea recta hacia el pastizal abierto. Como valor 
de importancia se emplea el número de individuos presentes de cada especie, por considerarlo más fiable que las estimaciones de cobertura, necesidad impuesta por el nivel al que se desarrolla el trabajo. Al ser todas las especies de pequeño porte se consigue una buena representación de la fisonomía del pastizal, además de la referida precisión en los resultados. Un marcado permanente de las unidades permitió completar los inventarios durante los meses de Mayo, Junio y Julio, anotándose las especies en el momento de mayor plenitud.

La profundidad del suelo (potencia edáfica) se midió por medio de perforaciones hasta llegar a la roca madre. Las profundidades pequeñas fueron estimadas cada $10 \mathrm{~cm}$. con el objetivo de conocer cualquier cambio más o menos pronunciado del gradiente; por encima de los $20-30 \mathrm{~cm}$. de espesor no se siguieron intervalos regulares, pero en todo momento se tuvo en cuenta que quedara bien delimitada la presencia de oscilaciones en espacios cortos.

\section{RESULTADOS Y DISCUSION}

Mediante la aplicación del análisis de correspondencias, el primer eje (Fig. 1) ordena las unidades (incluídas en círculos) de acuerdo con su profundidad, quedando representados, desde los valores más positivos a los más negativos, los tres niveles de espesor del suelo con los que a grandes rasgos puede definirse la transección. Dichos niveles se relacionan respectivamente con la zona de profundidad muy reducida (1-19), la de cambio rápido (20-28) y la de profundidad considerable (29-80).

Para el segundo eje se presenta la tendencia a separar en un extremo negativo las unidades relacionadas con la variación brusca en el nivel de suelo. Con sentido hacia los valores positivos se van disponiendo las muestras restantes, tanto más alejadas cuanto más difieren de estas características, de forma que llegan a superponerse las profundidades bajas con las elevadas.

En conjunto, para los dos ejes, la situación de muestras y parcelas adquiere la forma de $\mathrm{V}$, resultado habitual cuando se examinan gradientes con un alto grado de perfección. Los vértices vienen a coincidir con los tres grupos establecidos (se señalan en la gráfica mediante trazos contínuos), si bien van quedando aisladas algunas agrupaciones intermedias o de contacto que marcan una trayectoria gradual. Con el propósito de 


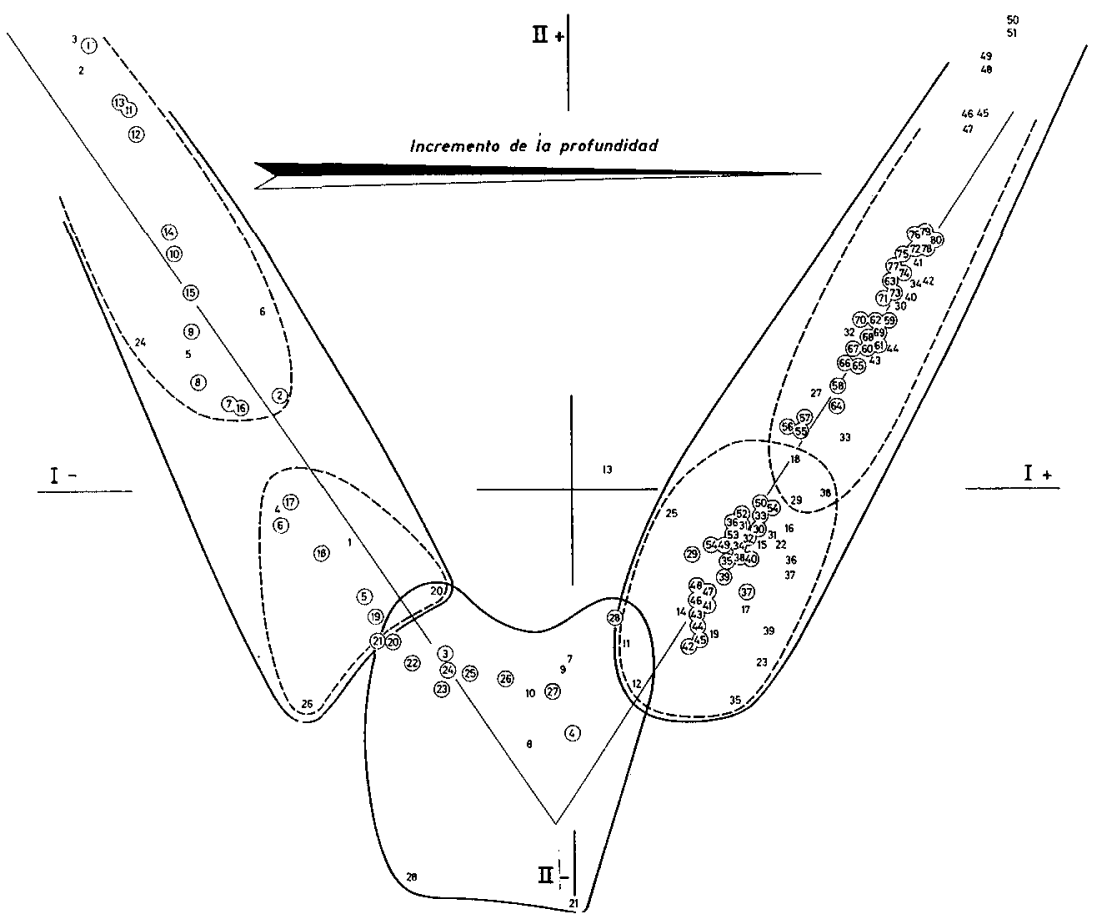

Figura 1.- Transección D. Situación de unidades y especies en el plano definido por los dos primeros ejes del analisis de correspondencias con datos de densidad.

\section{Tabla 1.-}

ESPECIES CUYA NUMERACION APARECE EN LA FIGURA 1

1.- Poa bulbosa L.

2.- Sedum arenarium Brot.

3.- Spergularia purpurea (Pers.) G. Don fil.

4.- Hypochaeris glabra L.

5.- Scleranthus annuus $\mathrm{L}$.

6.- Merendera pyrenaica (Pourret) P. Fourn.

7.- Ctenopsis delicatula (Lag.) Pounero

8.- Trifolium strictum L.

9.- Chamaemelum mixtum (L.) All.

10.- Molineriella laevis (Brot.) Rouy

11.- Moenchia erecta (L.) P. Gaertner, B. Meyer \& Scherb.

12.- Aphanes arvensis $\mathrm{L}$.

13.- Vulpia bromoides (L.) S. F. Gray 
14.- Spergula arvensis $\mathrm{L}$.

15.- Centaurea ornata Willd.

16.- Trifolium striatum $\mathrm{L}$.

17.- Ornithopus perpusillus L.

18.- Leontodon taraxacoides (Will.) Mérat

19.- Parentucellia latifolia (L.) Car.

20.- Erodium botrys (Cav.) Bertol.

21.- Myosotis discolor Pers.

22.- Anthoxanthum aristatum Boiss.

23.- Montia fontana $\mathrm{L}$.

24.- Logfia minima (Sm.) Dumort.

25.- Rumex angiocarpus Murb.

26.- Trifolium arvense $\mathrm{L}$.

27.- Teesdalia coronopifolia (J.P. Bergeret) Thell.

28.- Aira caryophyllea L.

29.- Echium vulgare $\mathrm{L}$.

30.- Trifolium subterraneum $\mathrm{L}$.

31.- Eryngium campestre L.

32.- Taeniatherum caput-medusae (L.) Nevski

33.- Trifolium micranthum Viv.

34.- Agrostis castellana Boiss. \& Reuter

35.- Narcissus bulbocodium L.

36.- Hypochaeris radicata L.

37.- Juncus capitatus Weigel.

38.- Ornithopus compressus L.

39.- Linaria elegans Cav.

40.- Tuberaria guttata (L.) Fourr.

41.- Trifolium dubium Sibth.

42.- Crepis capillaris (L.) Wallr.

43.- Cerastium glomeratum Thuill.

44.- Bellardia trixago (L.) All.

45.- Bromus hordeaceus L.

46.- Jasione montana L.

47.- Lepidium campestre (L.) R. Br.

48.- Anthemis arvensis L.

49.- Plantago lanceolata $\mathrm{L}$.

50.- Ranunculus paludosus Poiret

51.- Anthyllis lotoides L.

simplificar el esquema, dicha trayectoria se ha trazado según las rectas de regresión de las unidades negativas respectos al eje $\mathrm{I}(\mathrm{y}=-1.14-1.46 \mathrm{x}$, $\mathrm{r}=-0.94)$ y de las positivas de acuerdo con el mismo eje $(\mathrm{y}=0.97$ $+1.54 \mathrm{x}, \mathrm{r}=0.99$ ), aunque en las primeras se nota un cambio de dirección aproximadamente a partir de la unidad 20 . 
Conviene resaltar que las unidades características por su poco suelo $(1,13,11,12,14,10,15,9,8,7,16$ y 2$)$ suponen la existencia de condiciones rígidas, que actúan en el sentido de reducir el número de especies de posible asentamiento, y aún dentro de ellas son prcas las que presentan adaptaciones que las lleven a una mayor abundancia que en otras partes. En consecuencia, las especies representativas de estos tramos muy pobres participan con un tanto por ciento pequeño en el total inventariado, $y$, por otra parte, en algunas ocasiones son circunstanciales, dependiendo del tipo de pastizal que se establezca como comparación.

La unidad 4 aparece desplazada en dependencia con un ligero pero brusco ascenso de la potencia edáfica. Esto conlleva a su vez un arrastre de las unidades colindantes, particularmente de la 3 , pero también de la 5 y de la 6. Se trata de rupturas a situaciones de «stress», que tienen una respuesta acentuada en la vegetación. Así el pequeño subgrupo formado por $6,5,17,18$ y 19 obedece en realidad a causas similares. Para las dos primeras su diferenciación se debe a la causa citada mientras que los tres últimos forman la base a partir de la cual empieza el tramo de incremento rápido de la profundidad.

Como se ha indicado, en la parte negativa más distante del eje II aparecen las unidades $20,21,22,23,24,25,26,27$ y 28 que, junto con 3 y 4 , forman una secuencia progresiva ante el cambio brusco de la profundidad. El inicio de esta secuencia está constituído por $20,21,22$ y 3 , y enlaza con la zona intermedia anteriormente descrita.

Desde la muestra 29 a la 54 , ambas inclusive, se admite la posibilidad de formación de un subgrupo, que no constituye aún el pastizal típico, sino una zona de transición hacia él. Como zona de transición está sometida a varias influencias, lo que unido a sus treinta y cuatro o más centímetros de suelo motiva la aparición de un número considerable de especies. Por último (unidades 55 - 80), se entra en el pastizal típico, donde predomina con mucho la influencia del pastoreo. Es necesario resaltar que, aparte de la dependencia con la profundidad, existen otras causas como la distancia a la roca, o en zonas de características diferenciales patentes, que se superponen al efecto principal en la unidades de muestreo de considerable espesor de suelo.

En cuanto a las especies, su localización puede seguirse a partir de la tabla I. No obstante, pueden citarse como más representativas las siguientes: 
1) Zona de profundidad muy reducida.- Se define por las especies: Sedum arenarium, Spergularia purpurea, Logfia minima, Scleranthus annuus, Poa bulbosa, Hypochaeris glabra y Erodium botrys.

2) Zona de incremento brusco de la profundidad.- Definida por: $\mathrm{Mo}$ lineriella laevis, Trifolium strictum, Ctenopsis delicatula, Chamaemelum mixtum, Moenchia erecta y Aphanes arvensis.

3) Zona de profundidad considerable.- Subdividida a su vez en:

a. Zona de transición al pastizal.- Caracterizada por las densidades elevadas, entre otras, de Anthosanthum aristatum, Trifolium striatum, Parentucellia latifolia, Leontodon taraxacoides, Ornithopus compressus, Ornithopus perpusillus, Rumex angiocarpus, Spergulla arvensis y Centaurea ornata.

b. Zona de pastizal típico.- Con Agrostis castellana, Trifolium subterraneum, Bromus hordeaceus, Bellardia trixago, Crepis capillaris, Cerastium glomeratum, Trifolium micranthum, Trifolium dubium, Tuberaria guttata, Teesdalia coronopifolia, Anthyllis lotoides, Plantago lanceolata, Jasione montana, etc., que aportan características del pastizal estabilizado.

Como se desprende de los párrafos precedentes, el tamaño reducido de las unidades aplicadas tiene una validez que va en función de las especies dominantes. Estas constituyen una trama básica de altos valores de importancia que enmascara por completo la posible participación del azar en las consideraciones globales.

El que intervengan densidades elevadas es una premisa necesaria, porque los fragmentos metodológicos, cuando carecen de una base comparativa manifiesta, pueden conducir a interpretar las dispersiones establecidas por el análisis de correspondencias como efectos relacionados con unas causas tal vez inexistentes.

Volviendo a los ejes, en realidad la interpretación del II es sencilla. Si consideramos que el nicho espacial de una especie puede idealizarse -en un gradiente de interrelaciones perfectas - según una campana de Gauss, las muestras correspondientes a los extremos presentarán entre sí una similitud por defecto, diferenciándose de las centrales tanto más cuanto más se alejan de ellas.

La alta absorción de varianza del eje I (35.4\%) frente a los demás, 
pone de manifiesto la linealidad del gradiente. De aquí que sea aconsejable proceder a un análisis más preciso entre profundidad y coordenadas de dicho eje. La dependencia aparece reflejada con claridad en la figura 2, donde la distribución puntual de las unidades para las dos variables da un coeficiente de correlación $\mathrm{r}=0.91^{* * *}$, muy significativamente distinto de cero con una probabilidad del $99 \%$, siendo la ecuación de la recta $y=-1.61+0.05 x$.

No obstante, y debido a la casual distribución de las profundidades a lo largo de la línea muestreada, se observa que los datos no presentan una continuidad deseable, debiéndose este alto coeficiente más a la presencia de dos agrupaciones distintas que a una secuencia real. Por ello, se ha preferido considerar por separado cada uno de los niveles, con lo que se obtienen los resultados siguientes:

$$
\begin{array}{rll}
1-19 & \mathrm{y}=-2.01+0.096 \mathrm{x} & \mathrm{r}=0.78^{* * *} \\
20-28 & \mathrm{y}=-1.21+0.027 \mathrm{x} & \mathrm{r}=0.97 * * * \\
29-80 & \mathrm{y}=-1.33+0.049 \mathrm{x} & \mathrm{r}=0.62^{* * *}
\end{array}
$$

Aunque se produce una reducción esperada de los coeficientes -respecto al general y de acuerdo con sus grados de libertad-, todos siguen siendo sin embargo muy significativos.

Un aspecto más interesante lo constituyen las reacciones de las coordenadas del eje I ante las distintas profundidades. Así, a una respuesta rápida por debajo de los $20 \mathrm{~cm}$. (1 - 19), sigue una más lenta en la zona de cambio brusco en el espesor del suelo $(20$ - 28), para conservar unos valores intermedios entre los $34-45 \mathrm{~cm}$. de las unidades 29 - 80. La anomalía respecto a una tendencia global en la que estas reacciones deberían disminuir de una manera continua - los incrementos del suelo van representando un $\%$ menor del total- parece radicar, al menos en parte, en que la zona de variación acusada de la profundidad tiene una extensión muy pequeña que imposibilita en superficie sustituciones tan estrictas de las poblaciones inventariadas. Estas respuestas comparativamente más lentas de la cubierta vegetal, se traducen en una imprecisión de los límites, como se indica en la gráfica inferior de la figura 2 por medio de flechas.

En dicha gráfica, dada la fuerte correlación entre profundidad y coordenadas del eje I, cabría esperar que las curvas resultantes coincidieran en sus máximos y mínimos. Sin embargo, y además del desplaza- 

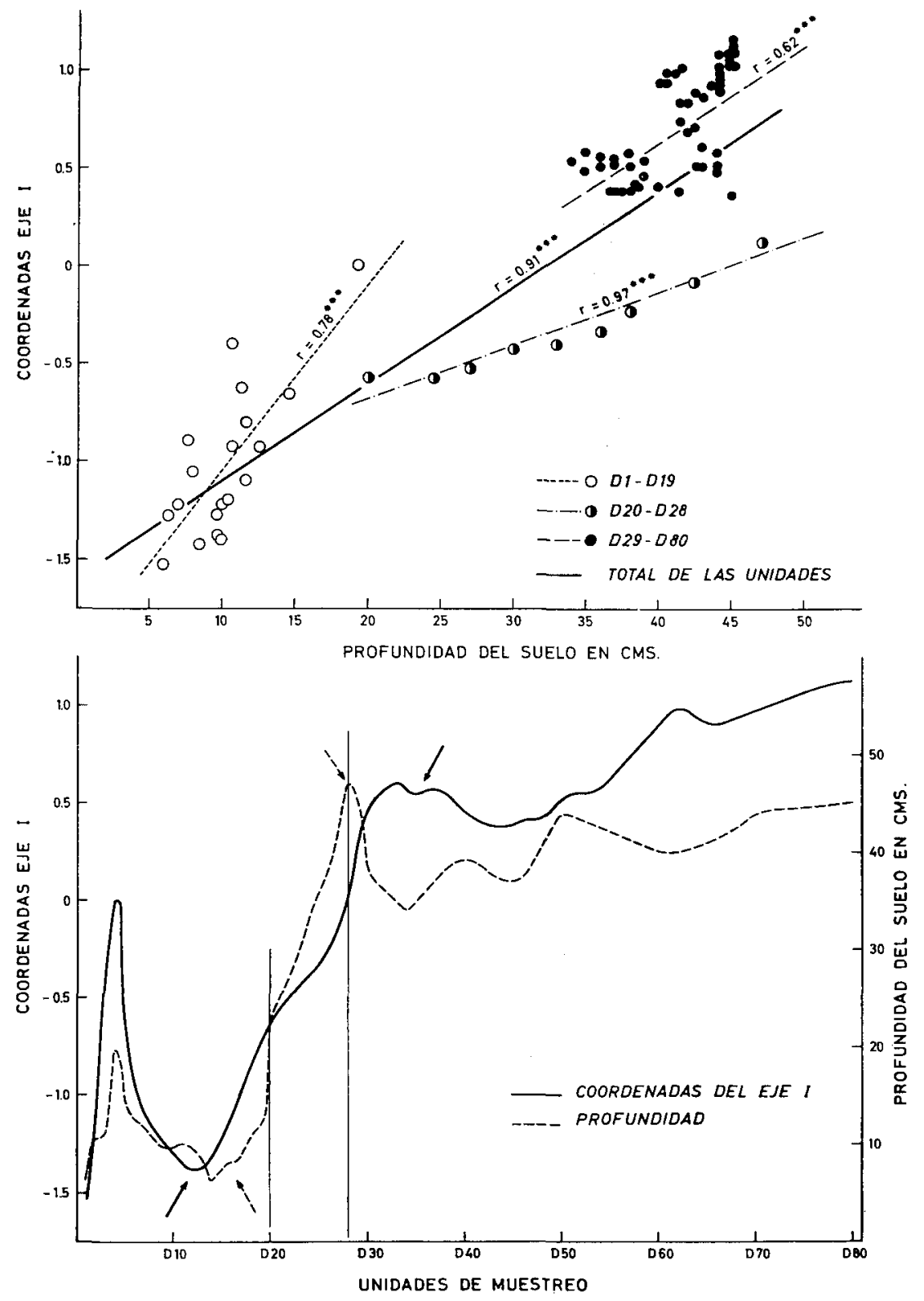

Figura 2.- Correlación entre el eje I del análisis de correspondencias y la profundidad del suelo. 
miento mencionado, ambas variables no parecen relacionadas desde 29 a 80, salvo en el contraste global de los grupos formados por 29-54 y 55-80 aproximadamente. El primero, más próximo a las condiciones de poco suelo e influenciado en gran manera por la zona limítrofe de cambio físico rápido (20-28), constituye una fase de contacto antes de que se produzca el ajuste de las especies típicas del pastizal. Su coincidencia con una capa de suelo algo menor con relación al segundo grupo, hace difícil conocer si la correlación observada $(r=0.62)$ depende en efecto de la profundidad o no es más que un artefacto casual debido a su lugar de asentamiento, pero es posible que ambos factores participen en cierta medida.

De aquí, que haya que insistir en valorar a la profundidad como el determinante general de las dispersiones que se producen sobre el eje I, pero no puede olvidarse que dentro de este aspecto amplio permanecen implícitos matices referentes tanto a la rapidez de variabilidad como al efecto causado por una mayor o menor cercanía a determinadas condiciones, que en definitiva se traducen en la necesidad de unos márgenes para que la diferenciación del nicho espacial de cada una de las especies pueda configurarse con precisión.

Los diferentes grupos sólo pueden comprenderse, en lo que respecta a sus especies, teniendo en cuenta la confluencia de una distribución espacial sometida a las imposiciones temporales. Esto hace que los trabajos muy concretos se vean en parte limitados, al ser representantes de unas coordenadas específicas de espacio y tiempo, y que únicamente cuando se cuente con un volumen suficiente de anotaciones similares podrá intentarse - a nivel de análisis factorial - la configuración de un esquema con validez general. Más factible es que en lo relativo a la ordenación y agrupaciones de las muestras, el modelo propuesto se proyecte con mayor amplitud en trabajos de esta índole, aún contando con el lógico cambio en la conformación de los ejes.

\section{BIBLIOGRAFIA}

BAZZAZ, F. A. (1968). Succession on abandoned fields in the Shawnee Hills, Southern Illinois. Ecology 49: 924-936. 
FREIJSEN, A. H. (1967). Some observations on the transition zone between the xerosere and the halosere on the Boschplaat (Terschelling, the Netherlands) with special attention to Centaurium vulgare Rafn. Acta Bot. Neer. 15: 668-682.

GASCO, J. M., CAÑADAS, M. E., GARCIA-SALMONES, R. y VERDEJO, A. (1979). Topografia del ricrorrelieve "gilgai» y su correlación con la vegetación en pequeñas áreas de la comarca de la Armuña, provincia de Salamanca (España). An. INIA, ser. general 6: 23-49.

PUERTO, A., RICO, M., LOPEZ FERNANDEZ, A. y FERNANDEZ HAEGER, J. (1981): Influencia de la topografía y de la profundidad del suelo en la ordenación de muestras procedentes de una comunidad de pastizal. Studia Oecologica II: 25-37.

SNAYDON, R. W. (1962). Micro-distribution of Trifolium repens and its relation to soil factors. J. Ecol. 50: 133-143.

ZEDLER, J. B. and ZEDLER, P. H. (1969). Association of species and their relationship to microtopography within old fields. Ecology 50: $432-442$. 